\title{
(6) OPEN ACCESS \\ Speak up-related climate and its association with healthcare workers' speaking up and withholding voice behaviours: a cross-sectional survey in Switzerland
}

- Additional material is published online only. To view please visit the journal online (http://dx.doi.org/10.1136/ bmjqs-2017-007388).

${ }^{1}$ Swiss Patient Safety Foundation, Zurich, Switzerland ${ }^{2}$ Institute of Social and Preventive Medicine (ISPM) University of Bern, Bern, Switzerland

\section{Correspondence to} Professor David Schwappach, Swiss Patient Safety Foundation, Zurich 8032, Switzerland; schwappach@ patientensicherheit.ch

Received 14 September 2017 Revised 5 February 2018 Accepted 25 February 2018 Published Online First 23 March 2018
Check for updates

To cite: Schwappach $D$ Richard A. BMJ Qual Saf 2018;27:827-835

\section{David Schwappach, ${ }^{1,2}$ Aline Richard ${ }^{1}$}

\begin{abstract}
Objectives To determine frequencies of healthcare workers (HCWs) speak up-related behaviours and the association of speak up-related safety climate with speaking up and withholding voice.

Design Cross-sectional survey of doctors and nurses. Data were analysed using multilevel logistic regression models Setting 4 hospitals with a total of nine sites from the German, French and Italian speaking part of Switzerland. Participants Survey data were collected from 979 nurses and doctors.

Main outcome measures Frequencies of perceived patient safety concerns, of withholding voice and of speaking up behaviour. Speak up-related climate measures included psychological safety, encouraging environment and resignation.

Results Perceived patient safety concerns were frequent among doctors and nurses (between 62\% and 80\% reported at least one safety concern during the last 4 weeks depending on the single items). Withholding voice was reported by $19 \%-39 \%$ of HCWs. Speaking up was reported by more than half of HCWs ( $55 \%-76 \%)$. The frequency of perceived concerns during the last 4 weeks was positively associated with both speaking up $(O R=2.7, p<0.001)$ and withholding voice $(O R=1.6, p<0.001)$. An encouraging environment was related to higher speaking up frequency $(O R=1.3, p=0.005)$ and lower withholding voice frequency $(\mathrm{OR}=0.82, \mathrm{p}=0.006)$. Resignation was associated with withholding voice $(O R=1.5, p<0.001)$. The variance in both voicing behaviours attributable to the hospital-site level was marginal.

Conclusions Our results strengthen the importance of a speak up-supportive safety climate for staff safety-related communication behaviours, specifically withholding voice. This study indicates that a poor climate, in particular high levels of resignation among HCWs, is linked to frequent 'silence' of HCWs but not inversely associated with frequent speaking up. Interventions addressing safety-related voicing behaviours should discriminate between withholding voice and speaking up.
\end{abstract}

\section{INTRODUCTION}

'Speaking up' of healthcare workers (HCWs) has raised considerable attention as an important resource for improving quality and patient safety during the last years. Speaking up is defined as assertive communication of patient safety concerns through information, questions or opinions where immediate action is needed to avoid patient harm. ${ }^{12}$ Several studies report positive associations of speaking up with patient safety. ${ }^{3-5}$ Examples of typical 'unsafe acts' in clinical settings that require speaking up are violations of hygiene protocols, medication administration errors or patient management decisions (eg, delayed transfer to the intensive care unit). In practice, speaking up can be very challenging and withholding voice is common among healthcare staff. Recent qualitative and quantitative research examined barriers for speaking up. The presence of audience (patients or relatives, coworkers), power dynamics, feelings of resignation and fears to damage relationships (with colleagues and superiors) have been identified as major reasons for withholding voice. ${ }^{1}{ }^{6-8}$ Authority gradients within the organisation or team strongly affect voicing behaviours. ${ }^{389}$ However, an individuals' decision to speak up or not is complex, dynamic and depends on the specific context and situation and, for example, previous speaking up interactions. ${ }^{10-12}$ Both, speaking up and withholding voice, are driven by motivational and intentional aspects. ${ }^{9}{ }^{13}$

Different approaches towards the conceptualisation of 'voicing behaviours' exist. In patient safety research, the terms 'speaking up' and 'withholding voice' are commonly considered as opposite poles of an unidimensional construct, that is, not speaking up is regarded equivalent to withholding voice and vice versa. 
However, withholding voice can be defined as an intentional behaviour not to verbalise ideas, information and opinions for the improvement of patient safety and is thus more than the absence of speaking up. Other disciplines such as organisational psychology and sociology have conceptualised speaking up and withholding voice as different dimensions of communication, allowing a more detailed insight in communication behaviour. Different forms of voice and silence can be discriminated along the content, the targets and the motives of voicing behaviours. ${ }^{14}$ For example, Dyne $e t$ al argue that employee silence and voice are best conceptualised as separate, multidimensional constructs and-based on the underlying motivesdifferentiate three types of silence (acquiescent silence, defensive silence and prosocial silence) and the three parallel types of voice (acquiescent voice, defensive voice and prosocial voice). ${ }^{13}$ In the context of whistleblowing and organisational deaf and silence, Jones and Kelly discuss that employees often send less formal and explicit voicing signals that are neither speaking up nor silence but that these signals often go unheard within organisations. ${ }^{15}$

Individuals' speaking up behaviours are influenced by personal (eg, age, personality), organisational (eg, hierarchy) and contextual factors, such as safety climate. $^{6} 1216$ Patient safety climate covers various aspects that are relevant for speaking up, such as psychological safety, leadership and teamwork climate. Manapragada and Bruk-Lee conceptualise 'organisational climate' as an explicit motive for employee silence about safety issues. ${ }^{17}$ However, the relationship between safety climate dimensions and speaking up behaviours is complex and not unambiguous. On the individual level, simulation studies present conflicting evidence whether trainee's speaking up behaviours towards their supervisors can be manipulated by this supervisors' encouraging or discouraging communication dynamics before. ${ }^{18} 19$ On the organisational level, evidence regarding the relationship between unit climate, psychological safety and staff speaking up behaviours is somewhat inconclusive as well. ${ }^{720}$ While it is clear that the complex dynamics between organisational climate and an individuals' voicing behaviours are not yet fully understood, these findings have also to be interpreted in light of how the underlying concepts are defined, operationalised and measured. For example, voicing behaviours are directly connected to specific events that triggered safety concerns and are thus highly context-specific whereas measures of perceived climate and its dimensions are rather generalised, sometimes inert conglomerates of experiences, perceptions and expectations. Climate may or may not only affect whether and how concerns are voiced, but may also even render how safety threats and unsafe behaviours are observed. In a climate in which unsafe acts become routine deviances and are obviously accepted employees may not even recognise them as instances that could require speaking up. ${ }^{21}$ As a very simplified example, frequent episodes of speaking up by staff may indicate either 'poor patient safety' or a 'good safety climate' or both. In summary, while speaking up-related safety climate is a valuable concept, there are still many open questions regarding its associations with speaking up behaviours of staff. ${ }^{22}$

In healthcare, little is known about the prevalence of different types of safety-related voicing behaviours and the relevance of safety climate for these. The main aim of our study was thus to examine the relationship between HCWs safety-related speaking up behaviours and speak up-related climate, discriminating between voicing and withholding concerns. In a first step, we assessed the frequencies of safety-related voicing behaviours withholding voice and speaking up. Second, we studied the associations of speak up-related climate with speaking up and withholding voice behaviours.

\section{METHODS}

\section{Survey instrument}

We used a recently developed survey instrument which covers an individual's speaking up-related past behaviours and perceptions of speaking up-related climate (see online supplementary appendix). ${ }^{23}$

The frequency of speak up-related behaviours includes three scales addressing (1) the frequency of perceived safety concerns ('perceived concerns scale' with three items), (2) the frequency of withholding voice, that is, NOT to speak up in specified situations ('withholding voice scale' with four items) and (3) the frequency of speaking up ('speak up scale' SUS with four items). Response options for the items in these scales are anchored to 'in the last 4 weeks' and include 'never' (0 times in the last 4 weeks), 'rarely' (1-2 times), 'sometimes' (3-5 times), 'often' (6-10 times) and 'very often' (more than 10 times during the last 4 weeks). Thus, higher mean scale values (range: 1-5) indicate higher frequencies of past speaking up and withholding voice behaviours, respectively.

Speak up-related climate is assessed by 11 items organised in three subscales: (1) the psychological safety for speaking up scale (PSS with five items), (2) the encouraging environment for speaking up scale (EES with three items) and (3) the resignation scale (RES with three items). The answers are coded in a 7-point-Likert scale from 'strongly disagree with this statement' to 'strongly agree with this statement'. Thus, higher mean scale scores (range: 1-7) indicate higher levels of perceived psychological safety at workplace, higher levels of perceiving the workplace as encouraging speaking up and higher levels of resignation with speaking up, respectively. Detailed information about item development, item selection and about psychometric testing of the German version of the questionnaire is available in Richard et al's study. ${ }^{23}$ In brief, psychometric testing (ie, explorative factor 
analyses (principal component analysis), reliabilities of the explored scales (Cronbach's alpha) and interitem analysis) was performed and the alpha of the climate scale was considered good with 0.86 . Analysis of variance was used to assess known-groups validity. The Swiss-German-language survey was translated and back-translated into French and Italian by professional translators. Native bilingual speakers (one French/ German and one Italian/German speaker) working in the patient safety field considered the final version of the translated questionnaires as accurate after some minor revisions.

\section{Statistical analysis}

Descriptive statistics (means and percentages of items and scales) assessed the distribution of the data. For easier interpretation, responses to the behaviour items were also dichotomised. The rule for splitting values was not based on distribution of responses (eg, median split) but on theoretical considerations to represent reporting of the behaviour versus non-reporting of the behaviour: responses on the 1-5 frequency scales were split and recoded as ' 0 ' ('never in the last 4 weeks') or ' 1 ' ('at least once during the past 4 weeks', covering 'rarely', 'sometimes', 'often', 'very often'). Percentages of responders with reporting the behaviour at least once in the past 4 weeks are reported. Cronbach's alpha was calculated as a measure of internal consistency of scales. Multivariable logistic regression analyses were used to model HCWs reported speaking up and withholding voice behaviours as outcome. The sample median was used as the cut-off value ( $<$ medianvs $\geq$ median) to dichotomise withholding voice and speaking up behaviours as dependent variables. Based on our hypotheses, the three speaking up-related climate scales (encouraging environment, psychological safety, resignation), perceived safety concerns and respondents' personal characteristics (age, gender, clinical function, years of employment in the hospital) were included as independent variables. We computed $r_{\text {wg(j) }}$ using rectangular (uniform) null distribution over all climate items as an index of inter-rater agreement among respondents within hospital-sites to examine whether HCWs share perceptions of speak up-related climate within their organisation. ${ }^{24}$ The value of $\mathrm{r}_{\mathrm{w}}$ - -sometimes called 'climate strength'-for patient safety climate research has recently been emphasised. ${ }^{25}{ }^{26} \mathrm{r}_{\mathrm{wg}(\mathrm{i})}$ values between 0.00 and 0.30 indicate lack of agreement, between 0.31 and 0.50 weak agreement, between 0.51 and 0.70 moderate agreement, between 0.71 and 0.90 strong agreement or very strong agreement (values $>0.90$ ). ${ }^{27}$ Due to the hierarchical structure of data (individuals within hospitals), we used multilevel regression modelling. 'Level 1' comprised individual responders whereas 'level 2' consisted of the nine hospital sites. We started with an intercept-only model ('empty model' without explanatory variables) to compute the variance between clusters (ie, hospital sites) divided by the overall variance (intraclass correlation coefficient (ICC)). A non-zero ICC implies that the observations are not independent. Likelihood ratio tests were computed to compare standard logistic regression models against the multilevel models. To model the contextual effects of speak up-related climate, the aggregated speak up-related climate scales (mean over hospital-sites) were added as level-2 explanatory variables. A likelihood ratio test was conducted to compare random intercept (only the intercept is allowed to differ across clusters) and random coefficient models (where slope and intercept are allowed to vary across clusters). Variance inflation factors were computed to check for multicollinearity between the included level-1 variables. For all models, VIF values were $<2$ indicating that collinearity was negligible. The final regression model was used to predict outcome probabilities for various scenarios. For all analyses, $p<0.05$ was considered statistically significant (two-sided).

\section{Study population}

The survey was conducted in one paediatric university hospital and one general hospital located in the German-speaking part of Switzerland, in six sites of a general hospital in the French-speaking part and in one general hospital in the Italian-speaking part of Switzerland. Hospitals participated with several major clinical departments, that is, internal medicine, surgery, emergency and intensive care units and others. All medical staff, mainly doctors and nurses, were asked to participate and received a self-administered written questionnaire, either by official or by internal mail. Hospitals sent two reminders per email to the entire sample.

\section{RESULTS}

Of invited staff ( $\mathrm{n}=2965), 1085$ individuals completed the questionnaire (participation rate of $36.6 \%$; range between hospitals: 31\%-42\%). Responders with profession other than nurses or doctors or with missing data on their clinical function were dropped from this analysis yielding a final sample of 979 completed questionnaires. Of responders, $81 \%$ were female, $21 \%$ were doctors and their mean age was 40 years. Sample characteristics are provided in table 1. Having perceived patient safety concerns at least once during the last 4 weeks was reported frequently by doctors and nurses (between 62\% and $80 \%$ depending on the single items). Withholding voice at least once during the past 4 weeks was reported by 19\%-39\% of HCWs depending on the respective item. Speaking up at least once during the past 4 weeks was reported by more than half of the HCWs (55\%-76\%) (table 2).

Cronbach's alpha for the climate scales were 0.82 (psychological safety), 0.77 (encouraging environment), 0.65 (resignation) and 0.86 (total scale). In simple logistic regression, the total speak up-related climate scale $(O R=0.46, p<0.001)$, defined as the mean over all items of PSS, EES and RES (RES reverse 


\begin{tabular}{|c|c|c|c|}
\hline & & $n$ & $\%$ \\
\hline \multirow[t]{9}{*}{ Hospital site } & A & 134 & 13.7 \\
\hline & B & 23 & 2.4 \\
\hline & C & 67 & 6.8 \\
\hline & D & 290 & 29.6 \\
\hline & $E$ & 21 & 2.2 \\
\hline & $\mathrm{F}$ & 151 & 15.4 \\
\hline & G & 32 & 3.3 \\
\hline & $\mathrm{H}$ & 40 & 4.1 \\
\hline & । & 221 & 22.6 \\
\hline \multirow{3}{*}{$\begin{array}{l}\text { Hospital region of } \\
\text { Switzerland }\end{array}$} & German-speaking part & 511 & 52.2 \\
\hline & French-speaking part & 317 & 32.4 \\
\hline & Italian-speaking part & 151 & 15.4 \\
\hline Males, \% & & 186 & 19.1 \\
\hline Age, mean (SD) years & & 39.9 & 11.4 \\
\hline \multirow[t]{7}{*}{ Profession } & $\begin{array}{l}\text { Nurses in training, } \\
\text { nursing assistants }\end{array}$ & 169 & 17.3 \\
\hline & Nurses & 500 & 51.1 \\
\hline & Nursing experts & 55 & 5.6 \\
\hline & Head nurses & 50 & 5.1 \\
\hline & Resident doctors & 77 & 7.9 \\
\hline & Attending doctors & 52 & 5.3 \\
\hline & Senior and chief doctors & 76 & 7.8 \\
\hline \multirow[t]{4}{*}{ Clinical disciplines } & Internal medicine & 222 & 23.0 \\
\hline & $\begin{array}{l}\text { Operative and } \\
\text { perioperative care }\end{array}$ & 288 & 29.8 \\
\hline & $\begin{array}{l}\text { Emergency and intensive } \\
\text { care }\end{array}$ & 195 & 20.2 \\
\hline & Others & 260 & 26.9 \\
\hline \multirow{2}{*}{$\begin{array}{l}\text { Duration of employment } \\
\text { in this hospital }\end{array}$} & $\leq 10$ years & 584 & 60.0 \\
\hline & $>10$ years & 390 & 40.0 \\
\hline \multirow{2}{*}{$\begin{array}{l}\text { Working hours per week } \\
\text { of patient care }\end{array}$} & $<25$ hours & 290 & 29.9 \\
\hline & $\geq 25$ hours & 680 & 70.1 \\
\hline
\end{tabular}

coded), frequently perceiving concerns $(\mathrm{OR}=1.60$, $\mathrm{p}<0.001)$ and being a nursing expert $(\mathrm{OR}=0.47$, $\mathrm{p}=0.047)$ or a senior/chief doctor $\quad(\mathrm{OR}=0.31$, $\mathrm{p}=0.002$ ) were significantly associated with frequent withholding voice, that is, equal or above the median frequency of withholding voice. Contrary, only frequently perceiving concerns $(\mathrm{OR}=2.76, \mathrm{p}<0.001)$ and being a nursing expert $(\mathrm{OR}=3.22, \mathrm{p}=0.005)$ or a senior/chief doctor $(\mathrm{OR}=2.22, \mathrm{p}=0.041)$ but not climate $(\mathrm{OR}=1.00, \mathrm{p}=0.98)$ were significantly associated with frequent speaking up, that is, equal or above the median frequency of speaking up.

The level of inter-rater agreement in speak up-related climate ratings among staff from the same hospital ranged between $r_{w g(i)}=0.50$ and $r_{w g(i)}=0.90$ among hospitals indicating moderate to strong agreement in climate ratings of staff from the same hospital. The mean $r_{\text {wg(i) }}$ was 0.77 (SD 0.13). The ICC of the empty multilevel logistic regression model with frequent speaking up as outcome was 0.016 , indicating that the variance at the hospital level accounted for $1.6 \%$ of the total variance (LR test vs logistic model $\chi^{2}=0.0421$ ). The ICC of the empty model with frequent withholding voice as outcome was 0.001 , indicating that the variance at the hospital level accounted for $0.1 \%$ of the total variance (LR test vs logistic model $\chi^{2}=0.4339$ ). Likelihood ratio tests were conducted to compare random intercept with random coefficient models (where slope and intercept of the climate scales allowed to vary across clusters). All tests indicated that the simpler models fitted the data better.

Results of the final multilevel models are reported in table 3. Frequently perceiving safety concerns was strongly associated with both voicing behaviours, speaking up (OR 2.76, p<0.001) and withholding voice (OR 1.59, $\mathrm{p}<0.001)$. Higher scores on the encouraging environment scale were associated with a higher likelihood to report frequent speaking up (OR 1.25, $\mathrm{p}=0.005)$ and a lower likelihood to report frequent withholding voice (OR $0.82, \mathrm{p}=0.006$ ). A higher level of psychological safety was associated with lower likelihood to report high frequencies of speaking up (OR 0.78, p=0.024). Higher levels of resignation were associated with higher frequencies of withholding voice (OR 1.49, $\mathrm{p}<0.001$ ), but not speaking up. Nursing experts and senior physicians were more likely to report frequent speaking up behaviours (OR 3.51, p=0.005 and OR 2.21, p=0.058) and less likely to report frequent withholding voice (OR 0.43, $\mathrm{p}=0.035$ and OR 0.28, $\mathrm{p}=0.002$ ). The non-significance of the hospital-site mean climate scales in both models indicates that there is no contextual effect of speak up-related climate on voicing behaviours over and above the individual-level effects. The hospital-site level explained $1 \%$ of the variance in frequently speaking up and $0.1 \%$ of the variance in withholding voice and was not significant.

The joint effects of perceived encouraging environment (EES) and resignation (RES) levels on the probability of high frequencies of voicing behaviours for four professional groups are illustrated in figures 1 and 2 , keeping all other variables at their mean. As this simulation shows, even at moderate levels of EES and RES (both values fixed at 4), the predicted probability of reporting a high frequency of withholding voice lies above the virtual cut-off of $\mathrm{p}=0.5$ for all professional (and thus hierarchical) groups. Contrarily, at moderate levels of EES and RES, the predicted probability of high speaking up frequency is $\geq 0.5$ for all groups, except residents. Even at lowest levels of EES and RES, nursing experts are rather likely to report high frequencies of speaking up $(\mathrm{p}=0.61)$.

\section{DISCUSSION}

Although the importance of HCWs speaking up to improve patient safety is increasingly acknowledged in healthcare organisations, little is known about how prevalent safety concerns and voicing behaviours are among staff. We observed in our study that 
Table 2 Frequencies of reporting perceived concerns, withholding voice and speaking up for at least once in the past 4 weeks (dichotomised) by professional group ( $n=979$ with non-missing clinical function data)

In everyday work, it sometimes happens that things go wrong and risks to patients arise. This could be as a result of medication error, poor hand hygiene or missing documentation. Over the last 4 weeks, how frequently...

\begin{tabular}{|c|c|c|c|c|}
\hline & & \multicolumn{3}{|c|}{ Relative frequencies } \\
\hline & & Total (\%) & Nurses $(\%)$ & Doctors (\%) \\
\hline \multicolumn{5}{|c|}{ Perceived concerns (Cronbach's a Ipha $=0.77$ ) } \\
\hline PC1 & ... have you had specific concerns about patient safety? & 80.0 & 80.4 & 78.5 \\
\hline PC2 & ... have you observed an error which — if uncaptured—could be harmful to patients? & 61.9 & 61.7 & 62.4 \\
\hline PC3 & $\begin{array}{l}\text {... have often have you noticed that your workplace colleagues haven't followed } \\
\text { important patient safety rules, intentionally or unintentionally? }\end{array}$ & 65.8 & 68.5 & 55.6 \\
\hline \multicolumn{5}{|c|}{ Withholding voice (Cronbach's alpha $=0.71$ ) } \\
\hline WV1 & ... did you choose not to bring up your specific concerns about patient safety? & 33.4 & 34.4 & 29.9 \\
\hline WV2 & ... did you keep ideas for improving patient safety in your unit to yourself? & 39.0 & 41.1 & 31.2 \\
\hline WV3 & $\begin{array}{l}\text {... did you remain silent when you had information that might have prevented a safety } \\
\text { incident in your unit? }\end{array}$ & 18.8 & 19.8 & 14.8 \\
\hline WV4 & $\begin{array}{l}\text {... did you not address a colleague (doctors and/or nurses) if he/she didn't follow } \\
\text { important patient safety rules, intentionally or unintentionally? }\end{array}$ & 39.4 & 42.7 & 27.3 \\
\hline \multicolumn{5}{|c|}{ Speaking up (Cronbach's alpha=0.86) } \\
\hline SU1 & ... did you bring up specific concerns about patient safety? & 75.8 & 76.8 & 71.7 \\
\hline SU2 & ... did you address an error which—if uncaptured_—could be harmful for patients ? & 69.6 & 70.2 & 67.7 \\
\hline SU3 & $\begin{array}{l}\text {... did you address a colleague (doctors and/or nurses) when he/she didn't follow } \\
\text { important patient safety rules, intentionally or unintentionally? }\end{array}$ & 64.0 & 66.5 & 54.5 \\
\hline SU4 & $\begin{array}{l}\text {... did you prevent an incident from occurring as a consequence of bringing up specific } \\
\text { concerns about patient safety? }\end{array}$ & 54.5 & 56.4 & 47.3 \\
\hline
\end{tabular}

perceived concerns are frequent and both, speaking up and withholding voice behaviours are common and do coexist. Frequencies of patient safety concerns were slightly higher in our study than in a recent US study with 1800 residents and in our previous study conducted in nine oncology departments in Switzerland. ${ }^{9}{ }^{12}$ One may speculate that our sample is more sensitive towards potential patient safety threats. However, we cannot differentiate between the true prevalence of safety problems and their recognition by employees, which is affected by various factors, such as level of training and individual psychological factors. ${ }^{28}$ Our results reveal a strong and positive association of the frequencies of perceived concerns with both speaking up and withholding voice. In fact, this observed association between concerns and behaviours confirms the validity of our model. Only few studies assessed frequencies of speak-up behaviours relative to perceived concerns in real-life settings (ie, outside the simulation setting). Future analyses should shed more light on how perceived concerns relate to occurrences of safety hazards and whether frequencies of speaking up, withholding voice and of perceived concerns differ between clinical disciplines, between teamwork climate or relative to different leadership styles in the organisation.

The safety climate of an organisation has been shown to be a crucial aspect for patient safety. ${ }^{3}$ A summary report from the Health Foundation (UK) covering more than 100 studies came to the conclusion that there is better evidence on the link between safety culture and safety behaviours, such as error reporting, than on the impact on patient outcomes. ${ }^{29}$ In our study, we assessed speak up-related climate, a specific aspect of safety climate, and observed associations with safety-related communication behaviours, that is, speaking up and withholding voice. Our study is novel in that we investigated the association of safety climate on the individual and hospital level with different voicing behaviours, namely, voice and silence. On the individual level, the overall speak up-related safety climate was strongly associated with lower frequencies of withholding voice, but not with higher frequencies of speaking up, which indicates that, indeed, these two behaviours are independent of each other. Interindividual processes of evaluating benefits and costs may differ for speaking up and withholding voice. ${ }^{10}$ Our results suggest that the speak up-related climate affects these trade-offs. Multilevel analyses using the climate subdimension scales reveal that a non-encouraging environment and high resignation levels were strongly associated with higher levels of withholding voice at individual level, but not the hospital level. Thus, a positive speaking up-related climate perceived by the individual decreased decisions to remain silent, irrespective of perceptions shared by staff at the hospital level. A strong encouraging environment was positively associated with speaking up, as expected. However, contrary to our expectations, higher levels of psychological safety decreased the frequency of speaking up and were not significantly related to withholding voice. Several rationales may help to explain 
Table 3 Results of multilevel logistic regression analysis with frequent speak up (SUS) and frequent withholding voice (WVS) as outcomes

\begin{tabular}{|c|c|c|c|c|}
\hline \multirow[b]{2}{*}{ Variable } & \multicolumn{2}{|l|}{ Frequent speaking up* } & \multicolumn{2}{|c|}{ Frequent withholding voice* } \\
\hline & OR $(95 \% \mathrm{Cl})$ & $P$ values & OR $(95 \% \mathrm{Cl})$ & $P$ values \\
\hline \multicolumn{5}{|l|}{ Individual level variables } \\
\hline Frequency of perceived concerns (quantiles) & 2.733 (2.367 to 3.155$)$ & $<0.001$ & 1.586 (1.399 to 1.798$)$ & $<0.001$ \\
\hline Psychological Safety (PSS) & 0.784 (0.634 to 0.969$)$ & 0.024 & $0.839(0.685$ to 1.029$)$ & 0.091 \\
\hline Encouraging environment (EES) & $1.253(1.071$ to 1.466$)$ & 0.005 & $0.816(0.705$ to 0.945$)$ & 0.006 \\
\hline Resignation (RES) & $1.024(0.896$ to 1.170$)$ & 0.732 & 1.494 (1.309 to 1.705$)$ & $<0.001$ \\
\hline Female gender & 1.424 (0.871 to 2.327$)$ & 0.159 & $0.811(0.510$ to 1.288$)$ & 0.374 \\
\hline Age, years & $1.003(0.984$ to 1.023$)$ & 0.751 & $0.996(0.978$ to 1.015$)$ & 0.699 \\
\hline \multicolumn{5}{|l|}{ Clinical function (to base: Nurse in training) } \\
\hline Nurses & $1.360(0.852$ to 2.171$)$ & 0.198 & 0.891 (0.569 to 1.394$)$ & 0.613 \\
\hline Nursing experts & 3.507 (1.457 to 8.443$)$ & 0.005 & 0.426 (0.193 to 0.943$)$ & 0.035 \\
\hline Head nurses & $1.941(0.843$ to 4.470$)$ & 0.119 & $0.607(0.280$ to 1.314$)$ & 0.205 \\
\hline Resident doctors & 0.835 (0.409 to 1.705$)$ & 0.621 & $0.547(0.278$ to 1.076$)$ & 0.081 \\
\hline Attending doctors & $0.833(0.347$ to 2.003$)$ & 0.683 & $0.742(0.339$ to 1.624$)$ & 0.456 \\
\hline Senior/chief doctors & 2.214 (0.974 to 5.032$)$ & 0.058 & 0.284 (0.131 to 0.618$)$ & 0.002 \\
\hline Employment in this hospital $>10$ years & $0.829(0.545$ to 1.261$)$ & 0.382 & $0.683(0.457$ to 1.020$)$ & 0.062 \\
\hline \multicolumn{5}{|l|}{ Hospital-site level variables } \\
\hline $\begin{array}{l}\text { Psychological safety (PSS), hospital-site } \\
\text { mean }\end{array}$ & 1.396 (0.500 to 3.897$)$ & 0.524 & $1.018(0.458$ to 2.266$)$ & 0.965 \\
\hline $\begin{array}{l}\text { Encouraging environment (EES), hospital- } \\
\text { site mean }\end{array}$ & 0.886 (0.285 to 2.757$)$ & 0.834 & 0.971 (0.381 to 2.474) & 0.950 \\
\hline Resignation (RES), hospital-site mean & $1.380(0.258$ to 7.389$)$ & 0.707 & $1.072(0.322$ to 3.575$)$ & 0.910 \\
\hline McKelvey and Zavoina's R2 & 0.425 & & 0.359 & \\
\hline Overall model $p$ & $<0.001$ & & $<0.001$ & \\
\hline Intraclass correlation coefficient & 0.010 & & 0.001 & \\
\hline
\end{tabular}

*Positive outcome defined as frequency equal to or higher than the sample median, to base frequency below sample median.

EES, encouraging environment for speaking up scale; PSS, psychological safety for speaking up scale; RES, resignation scale; SUS, speak up scale;

WVS, withholding voice scale.

this counterintuitive finding: for example, it seems likely that subjects working in units with a high level of psychological safety may simply not perceive speaking up as a distinct and potentially invasive communication style but as a rather normal form of 'team working' and thus, report lower levels of speaking up even though they exchange concerns frequently. Another aspect which may play a role is that according to cognitive psychology, frequencies of an event are overestimated when they have a large emotional impact. ${ }^{28}$ In a working environment with low psychological safety, perceived concerns are probably accompanied by stronger emotional repercussions due to the non-supporting environment. These emotions may increase speaking up behaviours or reporting thereof. In addition, the negative association of psychological safety and speaking up may be directly linked to how these constructs were operationalised. The behaviours items are measured on a frequency scale asking for communicating behaviours towards coworkers and supervisors in specific situations. Psychological safety may not predominantly affect how frequently, but how and towards whom concerns are addressed. In addition, the definition of our outcome variables describes an individuals' communication behaviour relative to the behaviour of the collective. Psychological safety may be more important to render this collective behaviour rather than an individuals' degree of deviation from this. Future research is clearly warranted to understand the role of psychological safety in the context of voicing and withholding concerns. Such investigation may help to shed light on whether psychological safety affects speaking up behaviours or the memorising and reporting of them.

Our study confirms associations of clinical function, and thus hierarchy, with both types of voicing behaviours. ${ }^{369}$ We observed that nursing experts were more likely to speak up and less likely to withhold their voice compared with other professional groups. Nursing experts might be more aware of existing standards and guidelines, which may foster their speaking up behaviour. In their function as nursing experts, they probably also are more sensitive towards possible threats and rule violations and more used to provide feedback to other nurses. Nursing experts are higher in hierarchy compared with other nurses and nurses in training. Chief physicians had significantly lower frequencies of withholding voice compared with other 


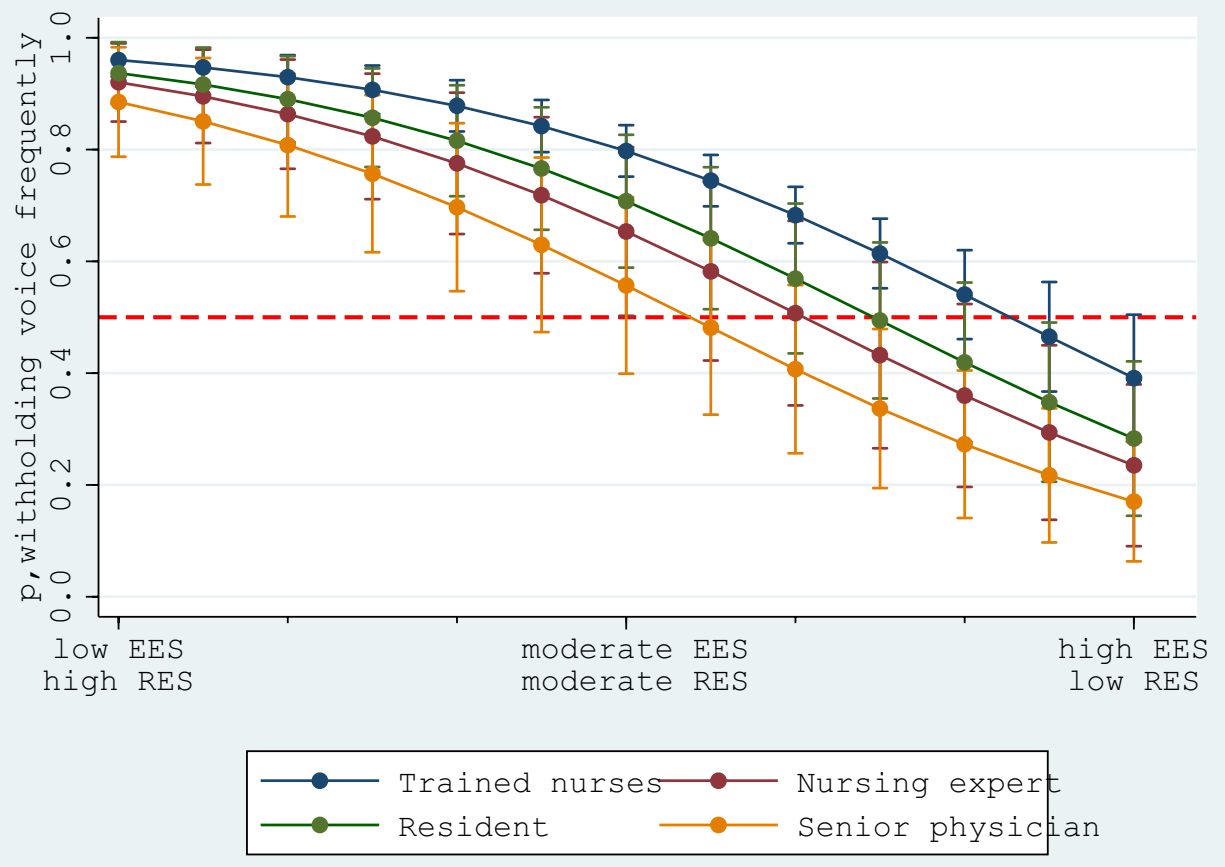

Figure 1 Predicted probability of reporting high withholding voice frequency ( $\geq$ the population median) relative to encouraging environment (EES, increasing values with range 1-7) and resignation levels (RES, decreasing values with range 1-7) by professional group, keeping all other variables at the mean. The dashed red line indicates the theoretical cut-off at $p=0.5$. EES, encouraging environment for speaking up scale; RES, resignation scale.

HCWs in our study, but there was no association with speaking up which seems plausible.

We found moderate to strong inter-rater agreement in speak up-related climate ratings of HCWs of the same hospital—with considerable variation in agreement between hospitals, that is, different levels of 'climate strength'. ${ }^{30}$ However, the fraction of variance in both voicing behaviours attributable to the hospital-site level was rather small. Importantly, hospital-site level climate ratings were not associated

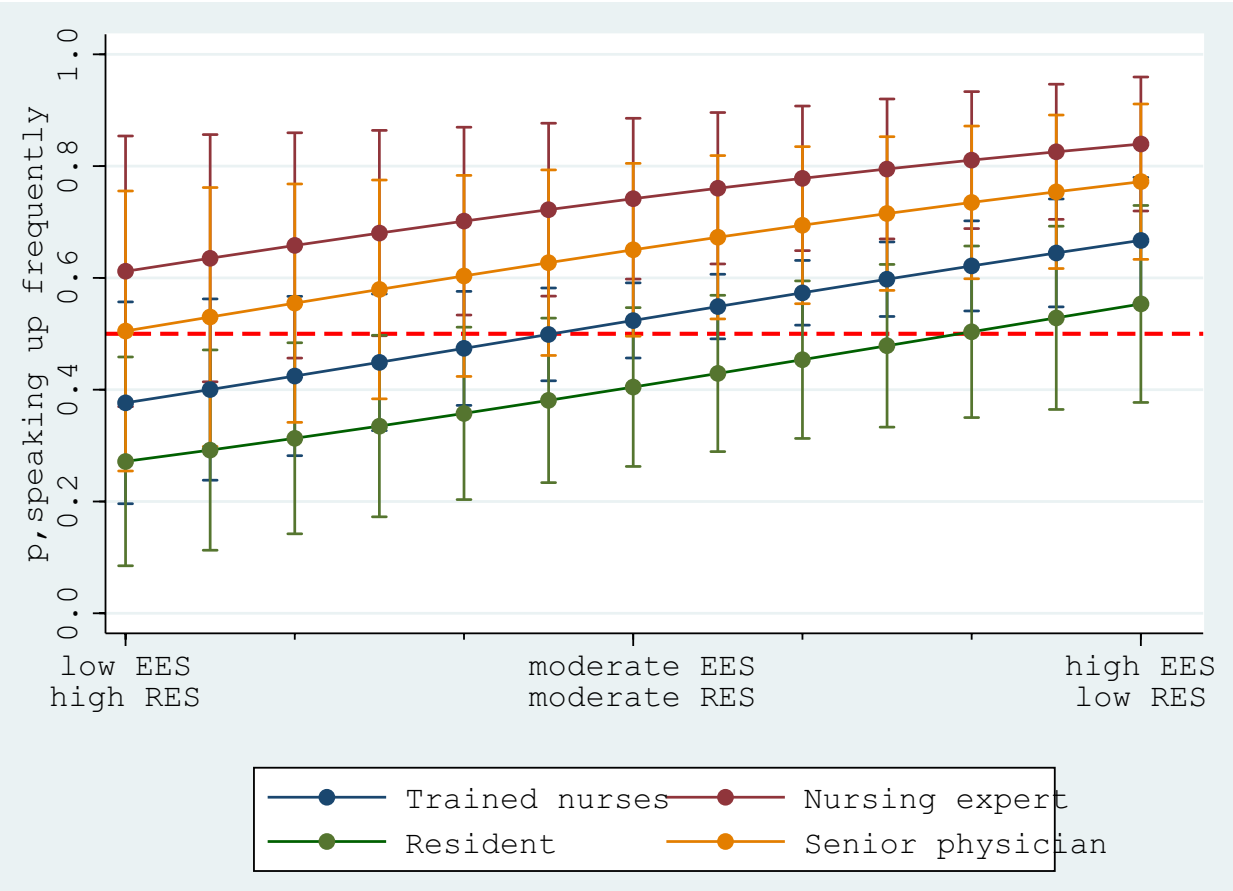

Figure 2 Predicted probability of reporting high speaking up frequency ( $\geq$ the population median) relative to encouraging environment (EES, increasing values with range 1-7) and resignation levels (RES, decreasing values with range 1-7) by professional group, keeping all other variables at the mean. The dashed red line indicates the theoretical cut-off at $p=0.5$. EES, encouraging environment for speaking up scale; RES, resignation scale. 
with frequency of voicing behaviours above and over the individual-level effects. In other words, it is the individuals' perception of climate that is relevant for expressing concerns and not the broader, shared climate 'context'. This can at least in part be explained by how our subdimensions of speak up-related climate are conceptualised and measured. For example, the encouraging environment scale explicitly asks whether the individual is encouraged to speak up by her supervisors and colleagues and whether she observes others speaking up. For individual trade-offs to speak up or to withhold voice, individual experiences of encouragement and the associated emotions seem to be of higher relevance, irrespective of whether others share the same experience.

This study provides novel insight into the relationship between safety climate and different types of voicing behaviours. However, several limitations have to be kept in mind. A main limitation of the study is the low response rate. We have no information about non-responders and, thus, it cannot be ruled out that our results are biased. We hypothesise that HCWs who do not speak up and withhold their concerns are more likely to be non-responders. Thus, speaking up behaviours and speak up-related climate may be overestimated whereas withholding voice behaviour may be underestimated. Other limitations origin in the self-reported nature of the data. Furthermore, reported speaking up and withholding voice behaviours may be subject to various biases, such that memories of past speaking up episodes may be more accessible and successfully retrieved compared with withholding voice events. As all of our data were obtained in the same survey, results may also be subject to same method bias. An important limitation is that we had no unit-level information from participants but only hospital-site affiliation to model the multilevel effect due to participant confidentiality. As safety climate research indicates that variance in climate is located rather on the microlevel (ie, unit or even ward) than the macrolevel of organisations (hospital), this may explain the lack of effect we observed on the hospital-level. ${ }^{3132}$ This view is strengthened by the variation we observed in inter-rater agreement of climate ratings across hospital-sites: where there is little agreement on climate on the hospital level, this supports the assumption that diverse microclimates exist. Future research including HCWs' unit-level or ward-level affiliation should investigate whether it is this microlevel climate that is important or whether speaking up behaviours are indeed relatively unaffected by climate above and over individual perceptions. Finally, our study is cross-sectional and we can thus draw no causal inferences. While it seems intuitive that climate affects withholding voice behaviour, more complex relationships with speaking up are likely. For example, positive speaking up experiences are likely to be the outcome of an encouraging environment and may themselves stimulate (re)-encouraging situations. Here, the climate would be an outcome of the speaking up event. Complex inter-relationships rather than a linear or one-way causal link between safety climate, safety behaviours and outcomes have been suggested previously. ${ }^{29}$

Despite these limitations, our results contribute to our understanding of HCWs' voicing behaviours. However, it is HCWs' speaking up that needs to be encouraged and also organisations' responsiveness to 'voice'. Our results highlight that resignationnegative experiences with or ineffectiveness of past speaking up episodes-is strongly related to withholding voice or 'silencing' staff. We believe that to achieve safety-oriented healthcare that values staff input as a vital resource to prevent harm, both are fundamental, empowering staff to speak up through encouragement and reinforcement and developing organisations' ability 'to hear' and respond adequately to voiced concerns.

Contributors DS and AR designed the study and interpreted the results. AR led the field phase. DS conducted the statistical analyses. Both authors approved the final manuscript.

Funding This study was funded by the Federal Office of Public Health, Switzerland (grant no. 15.002825).

Competing interests None declared.

Patient consent Detail has been removed from this case description/these case descriptions to ensure anonymity. The editors and reviewers have seen the detailed information available and are satisfied that the information backs up the case the authors are making.

Ethics approval The study was exempted from full ethical review by the Ethics Committee of the Canton of Zurich, Switzerland (BASEC-Nr. Req-2016-00462).

Provenance and peer review Not commissioned; externally peer reviewed.

Open access This is an open access article distributed in accordance with the Creative Commons Attribution Non Commercial (CC BY-NC 4.0) license, which permits others to distribute, remix, adapt, build upon this work noncommercially, and license their derivative works on different terms, provided the original work is properly cited and the use is non-commercial. See: http://creativecommons.org/licenses/ by-nc/4.0/

(C) Article author(s) (or their employer(s) unless otherwise stated in the text of the article) 2018. All rights reserved. No commercial use is permitted unless otherwise expressly granted.

\section{REFERENCES}

1 Schwappach DL, Gehring K. 'Saying it without words': a qualitative study of oncology staff's experiences with speaking up about safety concerns. BMJ Open 2014;4:e004740.

2 Lyndon A, Sexton JB, Simpson KR, et al. Predictors of likelihood of speaking up about safety concerns in labour and delivery. BMJ Qual Saf 2012;21:791-9.

3 Okuyama A, Wagner C, Bijnen B. Speaking up for patient safety by hospital-based health care professionals: a literature review. BMC Health Serv Res 2014;14:61.

4 Davenport DL, Henderson WG, Mosca CL, et al. RiskAdjusted Morbidity in Teaching Hospitals Correlates with Reported Levels of Communication and Collaboration on 
Surgical Teams but Not with Scale Measures of Teamwork Climate, Safety Climate, or Working Conditions. J Am Coll Surg 2007;205:778-84.

5 Robbins J, McAlearney AS, Scheck McAlearney A. Encouraging employees to speak up to prevent infections: Opportunities to leverage quality improvement and care management processes. Am J Infect Control 2016;44:1224-30.

6 Landgren R, Alawadi Z, Douma C, et al. Barriers of Pediatric Residents to Speaking Up About Patient Safety. Hosp Pediatr 2016;6:738-43.

7 Etchegaray JM, Ottosen MJ, Dancsak T, et al. Barriers to Speaking Up About Patient Safety Concerns. J Patient Saf 2017:1 (Published Online First: 4 November 2017).

8 Morrow KJ, Gustavson AM, Jones J. Speaking up behaviours (safety voices) of healthcare workers: A metasynthesis of qualitative research studies. Int J Nurs Stud 2016;64:42-51.

9 Schwappach DL, Gehring K. Frequency of and predictors for withholding patient safety concerns among oncology staff: a survey study. Eur J Cancer Care 2015;24.

10 Schwappach DL, Gehring K. Trade-offs between voice and silence: a qualitative exploration of oncology staff's decisions to speak up about safety concerns. BMC Health Serv Res 2014; 14:303.

11 Szymczak JE. Infections and interaction rituals in the organisation: clinician accounts of speaking up or remaining silent in the face of threats to patient safety. Sociol Health Illn 2016;38:325-39.

12 Martinez W, Lehmann LS, Thomas EJ, et al. Speaking up about traditional and professionalism-related patient safety threats: a national survey of interns and residents. BMJ Qual Saf 2017;26:869-80.

13 Van DL, Ang S, Botero IC. Conceptualizing Employee Silence and Employee Voice as Multidimensional Constructs*. J Manag Stud 2003;40:1359-92.

14 Brinsfield CT, Edwards MS, Greenberg J. Voice and silence in organizations: Historical review and current conceptualizations. Voice and silence in organizations. UK: BingleyEmerald Group Pub, 2009:3-33.

15 Jones A, Kelly D. Deafening silence? Time to reconsider whether organisations are silent or deaf when things go wrong. BMJ Qual Saf 2014;23:709-13.

16 Milliken FJ, Lam N. Making the Decision to Speak Up or to Remain Silent. Voice and silence in organizations. Emerald, Bingley: Ryan \& Oestreich, 2009:225-44.

17 Manapragada A, Bruk-Lee V. Staying silent about safety issues: Conceptualizing and measuring safety silence motives. Accid Anal Prev 2016;91:144-56.
18 Barzallo Salazar MJ, Minkoff H, Bayya J, et al. Influence of surgeon behavior on trainee willingness to speak up: a randomized controlled trial. J Am Coll Surg 2014;219:1001-7.

19 Friedman Z, Hayter MA, Everett TC, et al; Power and conflict: The effect of a superior's interpersonal behaviour on trainees' ability to challenge authority during a simulated airway emergency. Anaesthesia., 2015:70, 1119-29. doi.

20 Gilmartin HM, Langner P, Gokhale M, et al. Relationship Between Psychological Safety and Reporting Nonadherence to a Safety Checklist. J Nurs Care Qual 2018;33:1.

21 Edwards MS, Ashkanasy NM, Gardner J. Deciding to Speak Up or to Remain Silent following observed wrongdoing: The role of discrete emotions and climate of silence. Voice and silence in organizations. UK: BingleyEmerald Group Pub, 2009:83-109.

22 Ginsburg L. 'Speaking up' climate: a new domain of culture to measure and explore. BMJ Qual Saf 2015;24:661-3.

23 Richard A, Pfeiffer Y, Schwappach DDL. Development and Psychometric Evaluation of the Speaking Up About Patient Safety Questionnaire. J Patient Saf 2017.

24 James LR, Demaree RG, Wolf G. Estimating within-group interrater reliability with and without response bias. J Appl Psychol 1984;69:85-98.

25 Mascherek AC, Schwappach DLB. Patient safety climate profiles across time: Strength and level of safety climate associated with a quality improvement program in Switzerland-A cross-sectional survey study. PLoS One 2017;12:e0181410.

26 Ginsburg L, Gilin Oore D. Patient safety climate strength: a concept that requires more attention. BMJ Qual Saf 2016;25:680-7.

27 LeBreton JM, Senter JL. Answers to 20 Questions About Interrater Reliability and Interrater Agreement. Organ Res Methods 2008;11:815-52.

28 Tversky A, Kahneman D. Availability: A heuristic for judging frequency and probability. Cogn Psychol 1973;5:207-32.

29 The Health Foundation. Does improving safety culture affect patient outcomes? London 2011.

30 Vogus TJ. Safety climate strength: a promising construct for safety research and practice. BMJ Qual Saf 2016;25:649-52.

31 Campbell EG, Singer S, Kitch BT, et al. Patient safety climate in hospitals: act locally on variation across units. Jt Comm J Qual Patient Saf 2010;36:319-26.

32 Schwendimann R, Zimmermann N, Küng K, et al. Variation in safety culture dimensions within and between US and Swiss Hospital Units: an exploratory study. BMJ Qual Saf 2013;22:32-41. 